\title{
Héros et anti-héros ? Intérêts de connaissance et nouvelles rationalisations institutionnelles
}

Michel Lallement

\section{OpenEdition}

\section{Journals}

Édition électronique

URL : http://journals.openedition.org/ress/509

DOI : $10.4000 /$ ress.509

ISSN : 1663-4446

Éditeur

Librairie Droz

Édition imprimée

Date de publication : 1 décembre 2003

Pagination : 87-106

ISBN : 2-600-00912-4

ISSN : 0048-8046

Référence électronique

Michel Lallement, « Héros et anti-héros ? Intérêts de connaissance et nouvelles rationalisations institutionnelles », Revue européenne des sciences sociales [En ligne], XLI-127 | 2003, mis en ligne le 30 novembre 2009, consulté le 19 avril 2019. URL : http://journals.openedition.org/ress/509 ; DOI : $10.4000 /$ ress.509 
Michel LALLEMENT

\section{HÉROS ET ANTI-HÉROS? INTÉRÊTS DE CONNAISSANCE ET NOUVELLES RATIONALISATIONS INSTITUTIONNELLES}

La posture réflexive à laquelle invite toute discussion sur le mode de production des connaissances en sciences sociales n'est pas aisée à tenir. L'un des arguments les plus classiques qui explique ce nécessaire inconfort tient à la place singulière qu'occupe le sociologue dans le champ d'observation. L'effort qu'exige une investigation sociologique sur la sociologie peut se teinter de toutes les couleurs de l'objectivation, le discours n'en demeure pas moins «situé». Qu'il ait été travaillé dans des perspectives adorniennes, kuhniennes, foucaldiennes..., ce constat presque trivial n'a cessé de provoquer l'ire de tous ceux qui ont cru déceler dans une telle assertion un parti pris nécessairement anti-rationaliste. Les faiblesses du schéma ont été alors dénoncées de multiples manières pour condamner, pêle-mêle, un relativisme destructeur, une infalsifiabilité douteuse sur le plan épistémologique, une construction intrinsèquement aporétique, etc. Sans revenir dans le détail d'un débat ancien, récurrent et riche parfois de rebondissements originaux, il convient peut-être de rappeler que la critique n'émane pas toujours de là où on l'attend spontanément. C'est ainsi qu'E. Durkheim, pourtant couramment et abusivement taxé de «sociologiste», a consacré un cours entier en 1913-14 à défendre pied à pied une théorie rationaliste de la science en opposition à un pragmatisme qui se complaît à la dénonciation de l'arbitraire conceptuel et qui débouche surtout sur une forme d'utilitarisme logique qui pose problème à ceux qui ne se satisfont pas de l'attitude méthodologique «bon enfant» prônée par exemple par W. James. Pour faire tenir ensemble exigence rationaliste et point de vue sociologique sur la science, E. Durkheim conclut son cours de la façon suivante:

la vérité est une norme pour la pensée comme l'idéal moral est une norme pour la conduite (1955, p. 197).

Cette remarque introductive n'a pour autre ambition que de rappeler que, si périlleuse puisse-t-elle paraître, la posture réflexive ne condamne pas d'emblée au nihilisme ou à l'anarchisme épistémologique. Afin d'évaluer, de manière nécessairement partielle et partiale, le statut des sciences sociales contemporaines, je souhaite asseoir la réflexion sur une question de fond: à l'heure où se démultiplient les discours sur la fin des institutions (objet, par excellence, du savoir sociologique) et la disparition définitive de la société industrielle (qui a enfanté de la sociologie comme discipline constituée), à quel intérêt de connaissance peut-on (encore) lier la production du savoir sociologique contemporain? Pour avancer quelques éléments de réponse, je suivrai un fil directeur: celui du travail. Outre le 
fait que la sociologie du travail constitue ma spécialisation professionnelle, il est à ce choix deux raisons de fond. Premièrement, comme je le rappelle dans la suite de cette contribution, au sein d'une société industrielle pétrie de prométhéisme, le travail a longtemps fait figure de vecteur d'intégration sociale par excellence. Il est donc pertinent d'interroger le devenir de cette activité singulière en une période où les questionnements relatifs à l'insertion débordent généreusement hors des cadres étroits des cénacles académiques. Deuxièmement, le destin du travail et de la sociologie sont étroitement mêlés, si bien qu'interroger l'un éclaire immédiatement l'autre. Sans qu'il ne s'agisse là d'un propos novateur, C. Taylor (1998) a montré combien l'érection de la vie ordinaire (vie de production et de reproduction) au rang de «bonne» vie était caractéristique de notre modernité, et cela à la différence de périodes antérieures qui avaient relégué le travail et la famille au bas de l'échelle pour mieux valoriser la vita contemplativa, la guerre ou le politique. Sur les brisées des questionnements straussiens et arendtiens, les querelles récentes sur la fin du travail ont constitué une sorte de réminiscence à ces débats mais sans pouvoir persuader que l'avènement d'une «société de travailleurs sans travail » ait définitivement mis hors jeu le travail comme pratique et comme valeur centrales des sociétés développées. A la différence de ceux qui pressentent un déclin irrémédiable du travail, C. Taylor met en évidence ce paradoxe en vertu duquel, dans la Modernité,

\begin{abstract}
nous nous efforçons de maintenir une conception de ce qui est incomparablement supérieur tout en restant attachés aux intuitions modernes essentielles relatives à la valeur de la vie ordinaire. Nous sympathisons à la fois avec le héros et l'anti-héros; et nous rêvons d'un monde dans lequel nous pourrions être l'un et l'autre (Taylor, 1998, p. 41).
\end{abstract}

Ce sont les termes et les formes de résolution de ce paradoxe, ainsi que leurs conséquences épistémologiques pour les sciences sociales, qu'il m'intéresse de sonder ici. Pour ce faire, je commence par revenir sur le problème des intérêts de connaissance et je fais jouer ensuite, dans le champ actuel des sciences sociales du travail, la clef épistémologique forgée en 1965 par J. Habermas. Parce qu'elle est au cœur des préoccupations que je mets ainsi au jour, j'aborde en troisième lieu la thématique des institutions. L'objectif est de persuader que la construction d'un point de vue raisonné sur les institutions demeure une tâche centrale pour la sociologie contemporaine. C'est dans un tel esprit que, dans un quatrième temps, j'esquisse une rapide épure de quelques unes des forces à l'œuvre dans le processus de rationalisation institutionnel du travail qui accompagne ce que nous nommons désormais «modernisation réflexive». La dernière partie de ce texte fait retour sur l'épistémologie de la connaissance propre aux sciences sociales et, à la lumière des transformations évoquées précédemment, elle propose d'amender les termes de la grammaire habermassiennne élaborée au milieu des années 1960.

\title{
I. RETOUR SUR UN TRIPTYQUE
}

Commençons par cette question qui ne cesse de hanter nombre d'esprits: quelle est l'utilité des sciences sociales? Le point de vue le plus cynique peut aisément conduire à la dévalorisation la plus radicale tant il peut paraître absurde, au regard des questions majeures qui agitent le monde, de faire carrière et profession 
dans un univers où les moyens (la reconnaissance académique, le souci pécuniaire, le narcissisme médiatique...) l'emportent souvent sur la fin. Si la question paraît naïve, elle n'en demeure pas moins cruciale. P. Feyerabend l'a formulé avec son radicalisme habituel:

\begin{abstract}
Pouvons-nous continuer à financer des recherches qui ne servent qu'à un groupe réduit (mais en développement constant) de spécialistes, pouvons-nous continuer à écouter nos intellectuels alors que nous savons pertinemment qu'ils ne font que noyer des problèmes humains élémentaires sous des théories inutiles et remplacer la vie dans son ensemble par des modèles naïfs (marxisme, schémas évolutionnistes, etc.), est-il tolérable de continuer à vivre sous la domination d'un 'savoir' qui ne reconnaît pas les principaux motifs de paix - l'amour, la compassion, le sentiment du caractère sacré de la nature et de la vie naturelle - et porte donc sa part de responsabilité dans la dévastation de notre existence? Ou bien n'est-il pas de notre devoir d'informer tous les êtres humains des possibilités qui s'offrent à eux (notamment en matière de reconversion industrielle) afin qu'ils décident eux-mêmes de ce qu'ils souhaitent, en totale harmonie avec leur humanité? (Feyerabend, 2003, p. 140).
\end{abstract}

Je ne reviendrai pas ici sur les conditions sociales qui, au nom du scepticisme à l'égard de sciences réputées molles et partisanes, mènent régulièrement les sociologues à s'expliquer devant les tribunaux les plus divers : ceux de la raison scientifique, du politique, des médias... Je n'évoquerai pas plus l'existence des différents arguments - souvent largement convergents sur le fond - que mobilisent les sociologues pour dire à quoi sert, ou ne sert pas, leur science (Lahire, 2002). Pour répondre à la question posée en introduction, je vais plutôt emprunter à la tradition critique dont l'on sait que le projet originel est précisément d'interroger les fondements sociaux et épistémologiques des pratiques scientifiques (Horkheimer, 1978).

Plutôt que d'opérer un long détour par la théorie critique en son ensemble, je préfère utiliser le texte de la leçon inaugurale de J. Habermas à l'Université de Francfort en 1965, texte repris dans La Technique et la science comme idéologie et abondamment enrichi dans Connaissance et intérêt. On se souvient peut-être que J. Habermas inscrit largement ses propos dans le cadre de la querelle du positivisme qui anime la sociologie allemande au cours des années 1960 (Adorno, Popper, 1979). Mais, ce faisant, J. Habermas ne fait pas que batailler contre le positivisme poppérien. Il prend aussi ses distances avec le discours critique d' $\mathrm{H}$. Marcuse. J. Habermas s'écarte des perspectives tracées par ce dernier et distingue deux rationalisations. La première, qui correspond pleinement à la rationalisation formelle de type wébérienne, gouverne habituellement la science et la technique (adéquation presque aveugle des moyens aux fins); la seconde intègre explicitement des fins émancipatrices et se défait des risques d'aliénation au moyen de procédures délibératives de type communicationnelle. Dans le texte de 1965, J. Habermas entame la discussion en se plaçant sous les auspices de la critique husserlienne de l'objectivisme scientifique. Faut-il rappeler la conclusion de l'auteur de La crise des sciences européennes et la phénoménologie transcendantale? Après la décapitation de la philosophie par le positivisme,

nous sommes devenus conscients, au moins d'une façon très générale, que le philosopher humain et ses résultats n'ont nullement dans l'ensemble de l'existence humaine la simple signification d'un but culturel privé, ou limité d'une façon ou d'une autre. Nous sommes 
donc - comment pourrions-nous l'oublier? - les Fonctionnaires de l' Humanité (Husserl, 1976, p. 23).

Le problème est on ne peut mieux posé. Pour E. Husserl, seule la phénoménologie peut rompre avec l'attitude naïve et faussement objective si caractéristique des sciences européennes et cela au profit d'une attitude rigoureusement contemplative qui sait dissocier la connaissance de l'intérêt. L'auto-réflexion transcendantale est le garant d'une théorie pure: le philosophe doit son attitude théorique à une conversion qui l'affranchit des multiples «ondes fugitives» qui secouent notre monde.

L'intérêt de la position que défend J. Habermas est de ne pas opposer ceux qui, d'un côté, seraient capables de produire une théorie pure à tous ceux, objectivistes naïfs, qui ignoreraient la puissance des intérêts qui commandent la production de leur connaissance. Dans une tradition typiquement allemande, J. Habermas divise le champ des savoirs en trois registres de connaissances et y associe autant d'intérêts de connaissance spécifiques. Le triptyque est suffisamment connu pour que l'on n'y consacre pas moult commentaires. D'un mot, rappelons simplement que J. Habermas distingue les sciences empirico-analytiques dont l'intérêt est d'ordre technique, les sciences historico-herméneutiques dont l'intérêt est pratique et, enfin, les sciences praxéologiques et critiques dont l'intérêt est émancipatoire. Il peut paraître paradoxal de mobiliser ce modèle triadique de la connaissance dans la mesure où, dans la pensée du J. Habermas d'aujourd'hui, celui-ci est non seulement daté mais il souffre de plusieurs lacunes sur lesquelles je reviendrai en conclusion. La question du travail qui sert de fil directeur est par ailleurs peu traitée, à quelques exceptions près, dans l'œuvre habermassienne. La raison en est simple. Selon J. Habermas,

on ne saurait penser un 'véritable' agir communicationnel dans les activités soumises au système économique. Interaction et travail s'opposent, la pensée des interactions sociales devant se substituer au primat marxiste accordé au travail (Zarifian, 1999, p. 164).

En dépit d'une telle option, le modèle épistémologique forgé en 1965 me paraît doublement pertinent. Il offre d'abord un cadre heuristique pour poser à nouveaux frais le problème lancinant des intérêts de connaissance. La sociologie du travail des années 1960 (période pendant laquelle J. Habermas compose son propre texte) correspond assez bien ensuite à l'idéal-type des sciences praxéologiques fabriqué par le sociologue de Francfort. Il n'est donc pas illégitime de poser la question du positionnement actuel de cette spécialité, voire de la sociologie en règle générale, dans l'espace des intérêts de connaissance dessiné par J. Habermas ${ }^{1}$.

\footnotetext{
Je n'entrerai pas, par voie de conséquence, dans le cœur des débats qu'ont pu susciter l'épistémologie de la théorie critique en général et celle de J. Habermas en particulier. Sur ce point, les travaux de H. Albert (1987) demeurent un point de passage incontournable (voir notamment l'article «Herméneutique et science exacte: la problématique du sens et la question de la connaissance théorique» dans lequel $\mathrm{H}$. Albert met en évidence la parenté entre la problématique schelérienne des formes de savoir et la partition épistémologique des intérêts de connaissance proposée par J. Habermas).
} 


\section{TRAVAIL ET INTÉRÊTS DE CONNAISSANCE}

Les bilans sur ce que sont et ce que font les sciences sociales du travail ne font pas défaut aujourd'hui (Beckenbach, 1991; Gazier, 1991; Pillon, Vatin, 2003; Pouchet, 2001...). Plutôt que d'esquisser l'improbable synthèse d'une littérature pour le moins foisonnante, mon ambition consiste à sonder le socle épistémologique sur lequel reposent les principaux discours actuels ${ }^{2}$. De ce point de vue, le constat est sans appel: dans leurs pratiques comme dans leurs représentations, les sociologues (et les économistes du travail que j'associe pour l'occasion) n'émargent pas, dans leur grande majorité, au registre praxéologique de J. Habermas. On peut s'en persuader assez rapidement en parcourant les trois pôles décrits précédemment. Telle qu'elle est pratiquée sous les auspices du paradigme néo-classique standard, l'économie du travail doit être rangée, la première, dans l'ensemble des théories à prétention hypothético-déductive. Les économistes qui portent intérêt au travail de cette façon se demandent pourquoi le chômage existe et persiste, interrogent l'efficace économique de paramètres divers (règles administratives, coût du travail...) sur la régulation du marché du travail, proposent des instruments d'évaluation de l'action publique... En bref, ce sont d'abord des processus - pour ne pas dire des mécanismes - qui sont soumis à la question. Une telle approche se nourrit d'une épistémologie qui emprunte plus ou moins discrètement au modèle des sciences empirico-analytiques. La formalisation mathématique du raisonnement, l'appréhension causaliste qui prédomine dans le traitement des statistiques (ainsi qu'en témoigne le succès récent des modèles Logit), le recours à une démarche hypothético-déductive, la prétention nomologique et normative, l'adhésion rarement questionnée en tant que telle aux canons de l'épistémologie poppérienne... en sont autant d'indices pertinents.

On peut, pour s'en convaincre plus encore, citer cet extrait d'un petit manuel récent signé par deux économistes français qui font autorité dans le domaine:

d'une masse de faits, il est toujours possible d'extraire une situation particulière, choisie
à dessein, sans s'interroger sur son degré de généralité. La diversité des situations est
telle qu'il existe bien évidemment des chômeurs courageux, des chômeurs paresseux, des
chômeurs découragés, des chômeurs heureux, des chômeurs malheureux, etc. Dans ces
conditions, il est toujours possible de tendre un micro à monsieur Dupont ou à madame
Durand afin qu'il ou elle nous relate son expérience. Ces témoignages sont importants
pour comprendre et quelquefois ressentir une véritable empathie pour le vécu de nos
prochains, mais ils n'apportent aucune connaissance sur la situation de l'ensemble des
chômeurs, ni a fortiori sur les causes du chômage (Cahuc, Zylberberg, 2003, pp. 3-4).

On devine comment, par opposition, nos deux économistes pensent la science du travail qui est la leur:

Ce n'est pas en décrivant les expériences de quelques chômeurs que l'on peut comprendre les déterminants du chômage. Il faut, pour ce faire, élaborer des hypothèses permettant de bâtir une théorie, qui sera ensuite confrontée aux faits (ibid., p. 4).

Pour des raisons de commodité, je m'en tiendrai au cas des sciences sociales du travail françaises. Mais le diagnostic est de même nature, me semble-t-il, au-delà des frontières de l'hexagone ainsi que dans de nombreux autres compartiments spécialisés des sciences de l'homme et de la société. 
Cette profession de foi objectiviste bénéficie, en apparence du moins, de la rigueur logique à laquelle contraint la formalisation mathématique. Mais, à y regarder de plus près, l'on s'aperçoit vite que le positivisme qui sourde les propos ne saurait occulter certaines faiblesses intrinsèques parmi lesquelles figure en bonne place une ambition nomologique démesurée ${ }^{3}$. Ce talon d'Achille apparaît à l'évidence à l'examen de pratiques qui consistent à importer des schémas d'analyse forgés outre-Atlantique et à les appliquer tels quels sur le continent européen sans se soucier des conditions socio-historiques de leur production, et donc sans s'interroger sur la pertinence de leur mobilisation hors de l'espace social qui les ont vu naître. Le cas des modèles de contrat implicite mis en forme au milieu des années 1970 en convainc aisément ${ }^{4}$. Avec celle des salaires d'efficience, cette formalisation des relations de travail a connu un succès d'estime en France lorsqu'il s'est agi de trouver de nouveaux arguments théoriques pour dépasser les apories du paradigme walrasien. Mais, tandis que l'on célèbre bruyamment les vertus syntaxiques d'une théorie qui hypertrophie la rationalité individuelle aux dépens d'un marché que l'on reconnaît faillible, nulle voix s'élève pour en contester la portée sémantique. Ne doit-on pas constater pourtant que la modélisation en terme de contrat implicite est avant tout la rationalisation des stratégies de gestion de la main-d'œuvre en vigueur dans les grandes entreprises américaines? N'est-il pas problématique, dans ces conditions, d'ériger en théorie pure et universelle la modélisation de pratiques étroitement situées? L'interrogation s'applique de manière similaire à ces modèles comme celui du droit à gérer ou de la négociation efficiente qui ont pour ambition de formaliser les relations de travail. Ici aussi, l'empreinte nord-américaine est plus qu'évidente (absence de pluralité syndicale, négociation au niveau de l'entreprise, occultation du rôle de l'Etat...) mais rares, pour ne pas dire inexistants, sont les économistes du travail néo-classiques européens soucieux d'intégrer cette donnée idiosyncrasique dans le mode de traitement qu'ils proposent pour rendre raison des relations de travail de leur pays d'origine. Malgré ces faiblesses largement imputables à des intérêts d'ordre académique qui incitent à la confusion entre fins de connaissance et usage inflationniste de la formalisation, et en dépit d'innovations non négligeables, l'économie du travail néo-classique n'en continue pas moins de se déployer avec, en arrière-fond, une rhétorique épistémologique de type empirico-analytique.

La seconde balise de l'espace construit en 1965 par J. Habermas a un statut historico-herméneutique. Celle-ci nous renvoie vers un univers d'intellection qui assigne à la compréhension du sens la tâche centrale de répondre à un intérêt pratique, à savoir la "possibilité d'un consensus entre sujets agissant dans le cadre d'une conception de soi qui leur vient de la tradition» (Habermas, 1973, p. 149). A la condition d'associer cette perspective au vaste ensemble, aux phases et aux surgeons multiples, issu des travaux de D. Schleiermacher et de W. Dilthey (Apel,

Pour une revue critique de la «nouvelle» économie du travail néo-classique, cf. par exemple H. Zadjela (1994).

L'originalité des modèles de contrat implicite est d'analyser les relations de travail comme produit d'une entente intéressée entre un employeur et des salariés et, surtout, de leur appliquer des raisonnements utilisés par les théories de l'assurance. On peut, de la sorte, expliquer la rigidité salariale, l'existence du chômage... autant de phénomènes incompréhensibles dans le paradigme walrasien traditionnel. 
2000), alors nul doute que celle-ci structure une partie importante de la sociologie du travail française contemporaine. Pour être plus exact, un double souci caractérise les travaux qui, à un titre ou à un autre, gravitent autours de cette borne historico-herméneutique. Pour des raisons contingentes, le premier tient à l'introduction du temps dans l'analyse du travail et de l'emploi. A l'heure du chômage et de la précarité, l'on s'est progressivement aperçu que les transitions professionnelles, les itinéraires d'insertion, les passages par l'intérim, les stages ou le chômage, la sortie d'activité, les carrières en entreprise... pouvaient révéler une pluralité de mondes vécus et de destins difficilement compréhensibles à l'aide des seules catégories administratives utilisées pour qualifier, à un moment donné, la situation occupée sur le marché du travail. En plaçant ainsi la question des temporalités au cœur du propos, les sociologues s'interrogent sur les conditions et les effets des enchaînements de statuts et d'emplois occupés sur, hors ou aux marges du marché du travail. Dans la foulée du Linguistic turn, l'analyse des récits, les histoires de vie, le travail sur la narration... acquièrent un franc succès ainsi qu'en témoignent aujourd'hui les nombreux manuels destinés à frotter l'apprenti sociologue à ces méthodes singulières d'enquête du social. Mais l'attention portée au récit ne se comprend pas non plus hors d'un contexte intellectuel qui a consacré le retour en grâce des approches interactionnistes et des questionnements centrés sur l'identité.

On peut, pour cerner rapidement les modalités et les enjeux de cette (re)découverte herméneutique, emprunter la dichotomie mêmeté/ipséité qui sert à P. Ricœur pour définir l'espace de sens dans lequel se configure l'identité narrative. Au même qui correspond à une logique de structuration invariable dans le temps s'oppose l'ipséité (être ou chose en question) dont la construction passe non par la permanence mais par l'altérité. La question que pose alors P. Ricœur est la suivante:

l'ipséité du soi implique-t-elle une forme de permanence dans le temps qui ne soit pas réductible à la détermination d'un substrat, même au sens relationnel que Kant a assigné à la catégorie de substance, bref, une forme de permanence dans le temps qui ne soit pas simplement le schème de la catégorie de substance? (Ricœur, 1990, p. 143).

Les positions tenues par P. Ricœur ne recouvrent pas exactement, loin s'en faut, celles défendues par les sociologues qui trouvent eux aussi dans la rationalisation narrative une vertu heuristique pour appréhender ce qu'être soi peut signifier (Bertaux, 1997; Demazière, Dubar, 1997; Ferrarotti, 1983... $)^{5}$. Mais elles indiquent clairement les données d'un problème qui continue de faire couler l'encre en abondance.

Aussi, bien que l'épistémologie qui sous-tend les sociologies des récits n'épouse pas étroitement les formes du moule historico-herméneutique, l'on peut

Telle qu'ils l'appliquent aux récits d'insertion de jeunes sortis de l'école et en quête d'emploi, la méthode promue par D. Demazière et C. Dubar (1997) vise une objectivation minimale du langage ordinaire grâce à l'articulation de la Grounded Theory de B. Glaser et d'A. Strauss avec la sémiologie narrative d'A. Greimas. L'objectif est, en d'autres termes, de mettre au jour la logique structurale qui préside à la construction de récits et, plus généralement encore, l'existence de «mondes socioprofessionnels» qui participent directement de la construction identitaire des individus. 
mesurer avec intérêt toute la distance avec la tradition néo-proudhonienne et néomarxiste qui a longtemps prévalu en sociologie du travail. Cette dernière a toujours incité à la défiance à l'encontre du discours des acteurs et à toute tentative de questionnement sur les modes de construction et de représentation du Soi. Est-ce le prix à payer pour une rupture qui ne s'achève pas pour autant aux confins de l'herméneutique? Toujours est-il qu'il est ici également de nombreux angles morts qu'éclairent difficilement les promoteurs des diverses «méthodes » disponibles sur le marché des récits de vie: chausse-trappe de l'illusion biographique, articulation problématique entre exploitation d'un entretien isolé et construction de types, hypertrophie du contenu de l'entretien aux dépens des conditions d'énonciation du discours produit par l'enquêté, etc. Mais qu'importe. Notons simplement pour clore ce point que si le retour du Sujet en sociologie est aussi multiforme qu'ambigu, l'un de ses effets, et non des moindres, est d'avoir donné à la parole et au récit des lettres de noblesse sociologiques que lui contestent toujours ceux qui, nourris au lait de l'épistémologie poppérienne, s'évertuent à produire une improbable science positive du travail et de l'emploi.

Il est temps d'aborder maintenant le dernier des espaces épistémologiques au sein duquel J. Habermas rangeait naguère les sciences sociales. Comme je l'ai déjà suggéré, la sociologie du travail des années 1960 et 70 partage de nombreux points communs avec les sciences praxéologiques et critiques, à commencer par ce souci d'œuvrer en faveur d'une émancipation collective des groupes dominés. Cette sociologie a longtemps focalisé son attention sur ce qu'elle considérait comme le noyau dur du monde de l'exploitation, à savoir l'atelier des grandes entreprises industrielles. Parmi les multiples caractéristiques de cette approche du travail, je retiens deux traits singuliers qui, au tournant des années 1960 et 70, participent directement de cet intérêt de l'émancipation. L'usage presque banal mais parfois confus de la notion d'aliénation tout d'abord: cette façon de lire le travail au prisme de la domination était avant tout au service de la dénonciation des méfaits de la division du travail dont le taylorisme représentait l'incarnation la plus parfaite et donc la plus abhorrée. Le second trait est cette croyance, qui nous paraît naïve aujourd'hui, dans les vertus émancipatrices des «nouvelles » technologies. Les espoirs fondés par S. Mallet (1963) dans la «nouvelle» classe ouvrière présente dans les secteurs les plus avancés du moment (électronique et électromécanique, pétrole, énergie électrique) en disent long sur cette volonté d'émancipation collective dont la sociologie du travail est à la fois le vecteur et l'écho.

Je ne pense pas trahir celles et ceux qui se reconnaissent toujours de cette filiation «critique » en caractérisant doublement leur posture et leurs travaux. Il s'agit, en premier lieu, de maintenir ferme la priorité donnée à l'analyse de l'action et de la situation de travail. Forte d'une réelle volonté d'accumuler le matériau empirique, la démarche mène toujours vers l'atelier avec cette interrogation majeure plantée au cœur de la problématique des recherches: le post-taylorisme n'est-il pas en fait qu'une simple réduplication de son prédécesseur immédiat et ne faut-il pas apercevoir là une nouvelle ruse de la raison capitaliste? Les réponses proposées sont en général plus subtiles que ne le laisse penser l'énoncé grossier que je viens de présenter. Certains soulignent ainsi le caractère asynchrone de la modernisation des entreprises (Linhart, 1994), d'autres pointent la pluralité des modèles nationaux de post-taylorisme (Durand, Stewart, Castillo, 1998), etc. Dans tous les cas cependant c'est toujours au nom de la distance critique et de la défense des 
intérêts des dominés que ces sociologues du travail ont vivement réagi à l'encontre de la sociologie de l'entreprise qui émerge au cours des années 1980. Cette dernière échappe effectivement aux canons classiques de la sociologie du travail en ce que son programme vise avant tout à reconnaître en l'entreprise non pas simplement un foyer opaque où brûle le feu de l'exploitation et de la domination mais une véritable institution au sens sociologique fort du terme ${ }^{6}$.

Au terme de la première étape qui vient d'être franchie, la conclusion qui s'impose est la suivante: dans un champs de connaissances où les enjeux d'émancipation ont été maintes fois soulignés, la grille de lecture épistémologique habermassienne échoue quand il s'agit de rendre raison de la multiplicité des approches du travail d'aujourd'hui. Il est deux façons de tirer parti d'un tel constat. La première invite à regretter amèrement que, pour des raisons qu'il importe peu d'examiner ici, les sciences sociales aient majoritairement déserté le registre critique et praxéologique. Une seconde interprétation, beaucoup plus pertinente à mes yeux, consiste à interpréter cet éclatement des intérêts de connaissance comme l'expression symptomatique d'un débat qui n'en finit pas de travailler la sociologie et les disciplines qui lui sont proches: celui des modes d'institution du social.

\section{CRÉPUSCULE DES INSTITUTIONS?}

Longtemps ignorée par les uns ou tenue, à l'inverse, pour une évidence de premier ordre par les autres, l'institution est de nouveau prétexte à innovation et à dispute. Nul n'ignore que derrière le vocable d'institution les différentes disciplines constitutives des sciences sociales ne rangent pas toutes les mêmes choses. En fait, l'institution a un statut similaire à celui du jeu de L. Wittgenstein: il est des «ressemblances de famille» qui ne trompent pas et qui, surtout, rendent illusoire toute tentative de délimitation. C'est pourquoi on me fera grâce de ne pas poser le pied sur l'infini territoire consacré aux définitions de l'institution et de ne pas décliner davantage par le menu les multiples oppositions et typologies «canoniques »: institutions primaires/secondaires (A. Kardiner), institutions régula-

\footnotetext{
En fait, outre celles que nous avons déjà mentionnées, de multiples branches (sociologies de la régulation sociale, de l'emploi, des professions, de l'action collective, de l'action publique appliquée au marché du travail...) ont germé sur le tronc de la sociologie friedmanienne d'aprèsguerre. Et peu d'entre elles, pour ne pas dire aucune, ne revendique ouvertement un intérêt de connaissance émancipatoire. La sociologie de l'emploi qui hérite pour partie, mais pour partie seulement, de préoccupations relatives aux différences de traitement des genres sur le marché du travail est certes fort sensible aux effets d'inégalité. Mais la sémantique marxiste et l'idéal d'émancipation sont absents de la rhétorique qui organise ses propos. Ajoutons que ceux qui, à l'instar de S. Beaud et M. Pialloux (1999), sont les plus prompts à assumer un discours qui vise à dénoncer les faits de domination qui pèsent sur le monde ouvrier ignorent largement, assez paradoxalement, les situations de travail pour donner priorité à la condition ouvrière lato sensu. Quant aux autres perspectives directement issues de la tradition friedmanienne, elles s'orientent soit vers une pratique sociologique pensée comme vecteur d'aide à la décision (Reynaud, 1989), comme maieutique de l'action collective (Touraine, 1966) ou encore, dans une tradition plus crozérienne, comme mode d'intervention au service de l'optimisation des régulations organisationnelles (Friedberg, 2001).
} 
tives/constitutives (J. Rawls), institutions programmées/spontanées (F. Hayek)... L'important est de constater que, en dépit encore une fois d'une entente imparfaite sur le signifié, l'institution est une thématique qui se joue allégrement des territoires épistémologiques constitués.

Qu'on en juge. Dans le cas de l'économie du travail, même les chercheurs qui recourent le plus volontiers à la formalisation mathématique ont fait leur deuil du commissaire priseur walrasien et nombreux sont ceux qui reconnaissent l'existence de dispositifs de coordination autre que le marché (que ce soit l'organisation, des conventions, des outillages cognitifs...) sans lequel il est impossible de penser logiquement les régulations du travail et de l'emploi. Fidèle néanmoins à ses préceptes épistémologiques de base, l'économie standard a su intégrer le fait institutionnel dans son portefeuille de connaissances et surtout, grâce à la théorie des jeux, lui reconnaître une logique et une fonctionnalité économiques (Walliser, 1989). Les sociologues qui ont, quant à eux, porté intérêt au récit, à l'analyse du langage, à l'identité, à l'expérience... présentent cette singularité d'avoir introduit la subjectivité au cœur d'un raisonnement sociologique qui a longtemps refoulé cette dimension aux marges de la discipline. Partant, la tentation est de diagnostiquer une crise voire un déclin des institutions (l'idée de «désinstitutionnalisation » résume commodément le propos) afin de mieux rendre raison des aventures des individus et de leurs difficultés à être eux-mêmes (Dubet, 2002). Qu'en est-il enfin de ceux qui, fidèles à la tradition friedmanienne, scrutent sans relâche les horizons du post-taylorisme? Tout comme les économistes de la régulation avec lesquels le rapprochement a été opéré il y a plusieurs années déjà (Boyer, Freyssenet, 2000), ils sont obligés eux aussi de prendre à bras le corps la question des institutions dans la mesure où l'un des résultats majeurs de leurs investigations comparatives est l'existence d'une variété de configurations sociétales au sein desquelles prennent forme les multiples visages de l'ordre productif post-taylorien.

Le thème des institutions et de leur devenir est ainsi, on le voit, largement transversal aux trois pôles recensés précédemment. Que l'on ne se méprenne pas pour autant. Non seulement, et je le répète à dessein, l'institution des économistes n'est pas nécessairement la même, en dépit de certains airs de parenté, que celle des sociologues ou encore que celle des politistes. Mais, surtout, le questionnement ne sonne pas de la même manière. Après avoir mis au ban de la science la tradition qui s'en réclame, les économistes (re)découvrent l'institution. Certains sociologues diagnostiquent quant à eux une défaillance des systèmes normatifs tandis que d'autres, qui émargent à la même corporation, ne savent trop que faire de la surabondance des arrangements institutionnels qui informent le travail d'aujourd'hui. Dans tous les cas cependant, une même inflexion est à l'origine de cet intérêt commun pour le fait institutionnel, celui d'une «grande transformation" des sociétés salariales modernes (Castel, 1995) dont l'un des effets, et non des moindres, est d'avoir obligé les sciences sociales à reconsidérer leurs postulats les plus fondamentaux. La croyance en l'efficience optimale de la régulation marchande est ainsi sérieusement ébranlée par l'existence d'un chômage durable mais aussi par l'échec répété des politiques de libéralisation et de flexibilisation à outrance des marchés du travail. La confiance dans les vertus intégratives des structures sociales des sociétés modernes se dérobe quant à elle à mesure que les identités perdent en cohérence, en homogénéité collective et en stabilité. Enfin, 
l'idée en vertu de laquelle une même matrice puisse, comme au temps glorieux de la civilisation industrielle, organiser les rapports de travail dans l'ensemble des pays développés se dissout d'autant plus rapidement que se banalise celle d'une diversité des mondes sociaux de l'entreprise et des formes nationales de posttaylorisme.

Comment s'étonner, dès lors, de l'importance des débats sur la place du travail au cours de ces dernières années? Nombreuses ont été les contributions qui ont interrogé la valeur d'une activité qui a servi de fondement à la construction des identités individuelles et collectives. C. Offe (1985) a été un des premiers à donner le «la» en constatant le décentrement du travail dans l'ensemble des intérêts de la vie et la moindre capacité de ce dernier à structurer la société. Ce questionnement qui n'engage pas que le travail (mais aussi l'école, la famille, la religion...) est indissociable de celui ouvert par U. Beck (2001) et A. Giddens (1994). La thèse de la modernisation réflexive que l'on trouve formalisée chez l'un et l'autre de ces théoriciens contemporains ne donne-t-elle pas raison à la sociologie de la « désinstitutionnalisation »? N'incite-t-elle pas au déploiement exclusif d'une sociologie de l'individu en vogue aujourd'hui et, finalement, ne condamne-t-elle pas aux ténèbres les efforts entrepris pour penser le fait institutionnel? Dans la mesure, en effet, où la posture réflexive qui caractérise les sociétés contemporaines engage à la prise de distance critique avec toutes formes d'explication par la contingence, l'individu est naturellement conduit au centre de la scène. Nombreux en sociologie sont ceux qui s'emploient activement à sonder les ressorts d'un processus d'individualisation dont l'on sait maintenant que, s'il provoque un appel d'air vers plus d'autonomie, de responsabilité, de liberté..., il accouche tout en même temps de pathologies multiples comme la «fatigue d'être soi» (Ehrenberg, 1998) ou encore la «culpabilisation des innocents» (Murard, 2003).

En fait, à lire attentivement les théoriciens cités précédemment, il apparaît que la réflexivité n'est pas qu'une affaire d'individus. Les institutions savent de mieux en mieux, elles aussi, intégrer des informations de nature diverses (dont les savoirs savants) pour améliorer leurs modes d'action et de régulation. Voilà pourquoi il n'est pas si paradoxal de constater que certains puissent annoncer le déclin des institutions tandis que d'autres en découvrent soudainement les vertus ou que d'autres encore en recensent la multiple variété. Le paradoxe s'épuise davantage dès lors que l'on considère que loin d'avoir dépéri, les institutions ont forci. A la différence d'hier simplement, elles sont désormais, «souples et décentralisées, [elles] deviennent des usines à fabriquer des individus plus autonomes et responsables » (Kauffman, 2002, p. 139).

\section{LES LINÉAMENTS D'UNE NOUVELLE RATIONALISATION INSTITUTIONNELLE}

Parce qu'elle éclaire l'état des débats actuels sur le statut de l'institution en sciences sociales et que, surtout, elle ouvre la porte à un vaste chantier à peine entamé aujourd'hui, il faut prendre au sérieux l'intuition livrée précédemment par J. C. Kauffman. Dans cet esprit, l'hypothèse que je fais mienne est que nous n'avons pas atteint le stade crépusculaire des institutions mais que, en revanche, nous assistons à une nouvelle rationalisation institutionnelle. La terminologie 
n'est pas fortuite. La modernité réflexive peut être utilement lue, en effet, au prisme du mouvement complexe et contradictoire de rationalisation du monde moderne décrit par $\mathrm{M}$. Weber. La modernité réflexive relève de ce point de vue à la fois de la continuité et de la rupture. Continuité si l'on considère que, sous couvert du vocable «rationalisation», M. Weber désigne cette capacité croissante dont sont dotés les hommes à exercer un contrôle conscient d'eux-mêmes. Rupture puisqu'un nouveau régime de régulation institutionnelle émerge sous nos yeux. Qu'en est-il de ce dernier? Comment celui-ci informe-t-il le travail, objet qui, jusqu'ici, a servi de fil conducteur à toute la réflexion? Sans prétendre à l'exhaustivité, je voudrais fournir quelques éléments de réponse et suggérer plus exactement que deux basculements méritent une mention particulière: celui de l'enrôlement axiologique en premier lieu, celui de la contractualisation du social en second lieu?

Le premier basculement d'importance a trait aux modes de mobilisation par et dans l'entreprise. Si l'on suit R. Sainsaulieu et D. Segrestin (1986), qui ont été les premiers à précipiter la discussion en ce sens, plusieurs facteurs auraient été propices à l'érection de l'entreprise comme institution centrale du social: la montée en puissance du souci économique, la «détaylorisation», des mobilités professionnelles contrariées par un chômage de masse, une mobilisation des salariés en faveur de l'emploi et de leur entreprise et, last but not least, la promotion de la culture comme outil de management. Cette nouvelle option managériale qui consiste à mobiliser par les croyances et les convictions n'est guère surprenante en une période où l'on découvre les limites d'un taylorisme débridé et où l'on s'aperçoit que l'entreprise est non seulement une organisation productive mais également un système à la fois culturel, symbolique et imaginaire (Enriquez, 1990). Dès lors que la mobilisation productive cesse de fonctionner sur les bases de la discipline et du respect des consignes pour laisser place à l'innovation et à l'initiative, l'intégration se dépouille de sa dimension instrumentaliste au profit de l'enrôlement axiologique. L'authenticité, la créativité, l'autonomie, la responsabilité... prennent statut d'injonctions qui peuvent d'autant plus aisément faire mouche qu'elles s'adressent à des salariés dotés d'un capital culturel peu comparable à celui de leurs alter ego d'hier. Ces invitations cachent mal, cependant, nombre de contradictions: exigence d'un contact plus personnel avec le public ou la clientèle mais évaluation des performances toujours calée sur des normes quantitatives, appel à l'innovation constante mais absence de moyens et de reconnaissance pour transformer le travail, réorganisation des activités sous couvert de polyvalence et durcissement en fait des vieilles pratiques tayloriennes, gestion asynchrone du changement (plus de participation formelle) mais maintien de la division du travail...

L'autocontrôle est, de ce point de vue, une illustration plus qu'exemplaire. Développée au cours de ces dernières années au sein de l'industrie automobile, le principe de base d'une telle pratique est l'attribution de la responsabilité du contrôle qualité à tous les opérateurs présents sur le process de production. Les ouvriers sont ainsi sollicités pour détecter et signaler immédiatement les

J'aurais pu également évoquer, entre autres illustrations, le thème des réseaux qui est au cœur des recompositions du capitalisme contemporain (Boltanski, Chiapello, 1999). 
défaillances du produit (principe de traçabilité) et cela afin que les retouches soient effectuées en temps réel. Le problème est qu'il s'agit d'une «responsabilité sous surveillance, menaçante, objet d'un encadrement strict» (Rot, 1998, p. 11). L'organisation souhaite garder la mémoire des incidents pour mieux s'en prémunir et, le cas échéant, repérer les coupables a posteriori. Ce faisant, elle oblige le groupe à «visibiliser» et à formaliser les fautes commises. Or, il n'est jamais simple de départager les responsabilités. Conséquence: les ouvriers contournent volontiers la règle formelle et

font leurs déclarations en empruntant des canaux informels d'action. De ce fait, ils rendent compte de leurs actes et tentent d'y remédier mais par des voies non institutionnelles. Ce compromis leur permet de se protéger tout en assurant la qualité (ibid., p. 19).

Fondée sur un enrôlement de type axiologique, cette responsabilisation des acteurs productifs est devenue pratique courante au sein des entreprises. Outre les nombreuses tensions et contradictions qu'elle produit, elle s'accompagne fort logiquement d'une reconfiguration des relations de travail qui, au modèle de la domination formelle, substitue celui de la contractualisation. En effet, dans la mesure où le cadrage cognitif et symbolique prend le pas sur «la commande par instructions et règlements qui caractérisait le modèle formaliste d'autorité», il «ne s'agit plus de fixer ex ante ce qu'il convient de faire, mais de déterminer les limites du négociable (ie les principes, les objectifs, les références que doit respecter une négociation)» (De Munck, 2000, p. 33). Voilà pourquoi l'entreprise est aujourd'hui pensée comme un nœud contractuel qui associe des parties prenantes dont les relations sont grevées par l'exigence de performance marchande. La contractualisation étant érigée en principe d'organisation externe (sous-traitance, franchise...) et interne (logique des centres de profit), il devient plus difficile que jamais de cerner les frontières de l'entreprise. Plus encore, et à l'instar de la mobilisation axiologique, la contractualisation des relations de travail se paye d'un surplus de dépendance au marché ou, si l'on préfère, d'un renforcement de l'étayage institutionnel sur lequel reposent les relations de travail. En effet, contrairement à l'interprétation juridique classique qui oppose institution et contrat, la contractualisation peut produire plus de domination, voire conduire à une «re-féodalisation du lien social» (Supiot, 2000).

\footnotetext{
La contractualisation ne signifie pas toujours indépendance relative des parties. On sait bien que certains contrats de sous-traitance accroissent les sujétions sur les entreprises qui les ont souscrits, créent des dispositifs stabilisant fortement les relations et introduisent des relations quasi-hiérarchiques entre les 'partenaires' (...) On peut donc dire que certains contrats créent de l'institution, au sens d'une stabilisation et d'une production forte de règle et d'autorité (Bernoux, 1999, p. 189).
}

De longs détours seraient nécessaires pour montrer comment, dans le champ des relations professionnelles, la «décentralisation» de la négociation relève pareillement d'une philosophie contractualiste dont, même partielle, la mise en application conduit paradoxalement à renforcer le pouvoir de régulation d'un acteur (celui de l'entreprise) au détriment des autres (salariés, syndicats, pouvoirs publics...) (Lallement, 2002).

Quoique le terme soit porteur de significations multiples et parfois fort confuses, la «procéduralisation » reste néanmoins le meilleur moyen de désigner les transformations de fond qui informent cette contractualisation des relations de 
travail. Cette évolution n'est pas réellement récente et elle est transverse à de multiples mondes sociaux et espaces nationaux. Dès les années 1960, N. Luhmann en avait déjà proposé une esquisse de théorisation. Dans La légitimation par la procédure, le sociologue allemand s'intéresse à trois types de procédures (électorales, législatives et parlementaires, judiciaires) afin de mettre en évidence l'épuisement du paradigme «classique» fondé sur la recherche d'une vérité pratique et sur le postulat que la «loi provient de la confrontation des opinions et acquiert ainsi une validité universelle au sens de vérité». Dans les sociétés modernes,

la vérité n'est plus que la transmission d'une représentation sur la base d'une certitude intersubjectivement contraignante et, sous cette forme, elle se distingue radicalement de l'adoption de représentations sur le fond d'une sympathie personnelle, de l'appartenance à un groupe ou d'une soumission au pouvoir (Luhmann, 2001, p. 15).

De là, N. Luhmann tire plusieurs conséquences qui demeurent plus que jamais d'actualité. Dans la mesure, d'abord, où la légitimation a perdu de son caractère transcendantal, l'horizontalité des rapports sociaux devient de plus en plus déterminante. Cela se traduit par des échanges et des négociations multilatérales, une place centrale accordée aux convictions subjective et, in fine, par le fait que le rapport à Autrui acquiert un statut de moralité. Dans la mesure ensuite où l'Etat joue un rôle différent, où les conventions se fragilisent, on assiste à une «juridicisation croissante » des rapports sociaux. La confiance devient, enfin, de plus en plus déterminante dans une société où l'intégration repose sur des compétences cognitives, des affinités électives peu stables... et non plus sur des solidarités à fort ancrage statutaire.

On peut vérifier assez aisément la pertinence de ces lemmes luhmaniens. Par exemple, la juridicisation des relations de travail est un fait maintenant avéré, qui n'est pas sans poser un problème de légitimité aux confédérations syndicales qui, en France, détiennent le monopole de la représentation légale des intérêts salariés. Ces organisations, dont le nom est gravé dans le marbre juridique par l'entremise de la reconnaissance de leur caractère représentatif, demeurent néanmoins le vecteur de la parole autorisée.

Ce n'est pas un hasard si, comme le remarque Benveniste, tous les mots qui ont un rapport avec le droit ont une racine qui signifie dire. Et l'institution entendue comme ce qui est déjà institué, déjà explicité, exerce à la fois un effet d'assistance et de licitation et un effet de fermeture et de dépossession» (Bourdieu, 1981, p. 4).

Sans même revenir sur une «crise» syndicale aux dimensions multiples, cet ébranlement d'une forme d'institutionnalisation de la régulation autonome a pour contrepartie l'invention et la diffusion de nouvelles rationalisations qui, entre autres conséquences, produisent l'éclosion de pathologies inédites. En raison des transformations de l'organisation et du marché du travail, le stress a ainsi pris le pas sur la lassitude, la souffrance et le harcèlement ont supplanté la thématique de l'aliénation tandis que l'exploitation est passée à la trappe de l'oubli au profit de la précarisation et de la désaffiliation.

Il ne s'agit pas là de simples jeux de mots mais de réels maux du Je. Le monde et le mode de vie des salariés ne sont plus structurés, du point de vue de leur avenir probable, par ces continuums de positions sociales constitutifs de la société salariale ou encore par ces maillages de postes et d'emplois qui servaient à ordonner 
les marchés internes du travail. A l'heure d'une flexibilité tous azimuts, les parcours dans l'emploi sont devenus chaotiques et incertains. La conséquence en est un bouleversement complet du sens à accorder à la notion de destin social:

\begin{abstract}
ce qui était le produit de déterminations sociales devient objet de choix et d'élaboration personnelle. Non seulement on peut, mais on doit aussi prendre des décisions ayant trait à la formation, au lieu d'habitation, au conjoint, au nombre d'enfants, etc. et toutes les sous-décisions s'y rattachent. Même dans les cas où le terme 'décisions' est excessif parce que l'on ne dispose pas d'alternative, l'individu doit 'assumer' les conséquences des décisions qu'il n'a pas prises (Beck, 2001, p. 290).
\end{abstract}

L'identité professionnelle «taylorienne» se construisait par ailleurs sur fond d'opposition «verticale» entre salarié et employeur et par l'entremise d'une gestion collective des conflits et des négociations. Avec les nouvelles rationalisations institutionnelles du travail - et notamment cette novation que constitue la relation de service - la prime est donnée à l'interaction avec le client, avec ses pairs, avec sa hiérarchie immédiate..., autant de sources potentielles de reconnaissance mais aussi d'ignorance et de mépris. Nul n'ignore en effet que

pour (se faire) coopter, séduire, pour se connecter, pour contractualiser, négocier, communiquer, il faut pouvoir assurer une bonne présentation de soi-même, savoir faire des concessions, s'ajuster à des milieux culturels changeants... (De Munck, 2000, p. 36).

Tel est loin d'être le cas de tous ceux à qui l'on demande aujourd'hui d'être les acteurs responsables de leur travail, de leur carrière, de leur retraite... C'est pourquoi la pression croissante du marché et celle, conjointe, de collectifs de travail de plus en plus interdépendants sont aussi au fondement du mal-être au travail ressenti par une partie de la population salariée. Teinté d'humiliations et d'offenses diverses (iniquités, peur, dépendance...), ce mal-être correspond bien à cette forme de mépris décrite par A. Honneth (2000) qui, aux côtés des violences physiques et des privations de droit, porte atteinte au Soi ${ }^{8}$. Il n'est pas incongru au total d'affirmer que la rationalisation réflexive est un facteur de dépendance accru à l'égard du marché en particulier et des institutions (formation, entreprise, consommation...) en général, non une grande transformation qui signerait le retrait de ces dernières hors de nos horizons de pratiques et d'intelligibilité'.

\title{
V. POLITIQUE DU SENS
}

Venant de toucher du doigt l'une des conséquences les plus pathogènes de la modernisation réflexive appliquée au champ du travail, je peux faire retour maintenant sur la question initiale posée en introduction à cette contribution: pour réfléchir sur le devenir des sciences sociales, les intérêts de connaissance tels

s Comme le remarque C. Taylor, «les psychanalystes ont souvent observé que les hystériques et les patients souffrant de phobies et de fixations, qui formaient le gros de leur clientèle à l'époque de Freud, ont fait place récemment à des patients qui se plaignent surtout de 'perte de l'ego', ou d'un sentiment de vide, de platitude, de futilité, de manque de raison d'être ou de perte d'estime de soi » (1998, p. 35).

9 U. Beck est d'ailleurs le premier à en convenir: «l'individualisation devient la forme de socialisation dépendante du marché, du droit, de la formation, etc., la plus poussée qui soit» (2001, p. 282). 
qu'ils ont été formalisés par la tradition allemande, et plus encore par la théorie critique, sont-ils toujours d'un grand secours? A cette question, deux éléments de réponse ont été apportés. A l'examen d'un cas singulier - celui des sciences sociales du travail - il apparaît d'abord que, pour des raisons qui tiennent autant à la dynamique des champs académiques qu'aux transformations structurelles des systèmes productifs, ces sciences revendiquent, au prix de l'éclatement intellectuel, l'occupation des trois registres épistémologiques distingués par J. Habermas. Deuxième élément de réponse: dans tous les cas de figure, la question de l'institution du social se pose avec vigueur et, avec elle, celle des fondements et des implications de la modernisation réflexive. Un rapide tableau des transformations majeures de ces deux dernières décennies convainc par ailleurs que, dans le champ du travail en tous les cas, l'on assiste moins à une dégénérescence institutionnelle qu'à une série d'évolutions tranchées aux accents parfois contradictoires: autonomie et dépendance accrues, rôle croissant de l'enrôlement axiologique, décentralisation et procéduralisation des régulations, surplus d'autonomie mais aussi fragilisation du lien social au risque du mépris et de la souffrance...

Quelles conclusions tirer, in fine, de ce détour par la modernisation réflexive? La première invite à réviser le schéma habermassien qui a servi de support à la présente réflexion. On trouve, chez J. Habermas lui-même, des objections aussi intéressantes que déterminantes. Dans la postface de 1973 de Connaissance et intérêt, J. Habermas reconnaît d'abord l'ambiguïté du concept idéaliste d' «autoréflexion». Il ne suffit pas de produire du savoir pour émanciper les individus et les sociétés des contraintes qu'ils s'infligent eux-mêmes. L'idée a gagné du terrain aujourd'hui :

la thèse, selon laquelle une meilleure connaissance de la vie sociale (même si cette connaissance est aussi empirique que possible) équivaut à un meilleur contrôle de notre destinée, est fausse. Elle est valable (et cela est démontrable) pour le monde physique, mais pas dans l'univers des phénomènes sociaux (Giddens, 1994, p. 50).

J. Habermas est conscient, par ailleurs, de l'imprécision des étalons de mesure à l'aune desquels il est possible de mesurer la «fausse conscience». En témoignent, de fait, les multiples apories rencontrées par la sociologie lorsque cette dernière a tenté, au cours des années 1960, de mesurer objectivement les formes et les niveaux d'aliénation au travail. Dans un texte récent, J. Habermas ajoute que

\footnotetext{
les formes classiques de l'idéologie ont perdu leur signification dans nos types de société : presque tous les journaux parlent aujourd'hui de la manière dont le capitalisme fonctionne et des modèles de répartition qu'il entraîne $\left(2003\right.$, p. 95-96) ${ }^{10}$.
}

\footnotetext{
Je laisse de côté la remise en cause, fort bienvenue du reste, de la thèse d'une histoire gouvernée d'une part par un processus d'autoproduction (où le travail occupe la place centrale) et, d'autre part, par un processus de développement culturel (fondée sur l'agir communicationnel): «j'aurais donc dû comprendre que la tentative visant à transposer le modèle freudien des névroses du domaine de la pathogenèse des individus singuliers à celui de la naissance et du développement des institutions sociales était vouée à l'échec (cf. p. 307: 'Ce sont les mêmes constellations qui poussent l'individu dans la névrose et amènent la société à établir des institutions'). A l'époque, j'étais fixé sur la critique de l'institutionnalisme fort, et surtout de la psychologie sociale d'Arnold Gehlen; c'est aussi pour cette raison que je me suis laissé entraîner dans une stratégie théorique qui tombait dans une opposition abstraite» (Habermas, 2003, p. 95).
} 
Par voie de conséquence, les sciences sociales ne peuvent plus revendiquer avec autant de succès qu'hier le monopole de la production d'un savoir authentique sur le monde social.

A quel enjeu doivent donc s'affronter les sciences sociales d'aujourd'hui et de demain? Je crois que l'intérêt de connaissance majeur reste irrémédiablement celui du sens, étant entendu qu'il convient de retenir ici une acception large du terme qui ne se réduise pas à sa seule dimension herméneutique. Le sens est, il est vrai, un thème immanent à l'histoire des sciences de l'homme et de la société. M. Foucault (1957) estime que la fin du XVII ${ }^{\mathrm{e}}$ siècle constitue le tournant décisif de la découverte. Les sentiers qui, depuis, nous ont mené au sens sont multiples. Ils ont été balisés par ces guides aussi prestigieux que S. Freud, M. Weber, E. Durkheim, M. Mauss, M. Heidegger, L. Wittgenstein, K. O. Appel, etc. Empruntés à l'envi, ces chemins ne méritent-ils pas d'être revisités avec en besace les multiples arguments de la modernisation réflexive? Assurément oui. Le problème est que les sentiers ne convergent pas vers un unique foyer d'intelligibilité. Aussi faut-il choisir. Le plus pertinent me semble être de continuer à marcher sur les brisées de l'école française de sociologie de façon à appréhender le sens de concert avec une intentionnalité, des pratiques et des croyances qui toutes s'étayent sur un lit de socialité. Comme le note justement V. Descombes, on ne peut s'en tenir aux apports des philosophies individualistes et structuralistes:

pour comprendre l'autorité de l'esprit objectif sur les sujets, il convient de concevoir tout à fait autrement la fonction du sens institué (impersonnel) dans la formation et la communication des pensées. La priorité de l'impersonnel sur le personnel n'est pas du tout comme la priorité du texte sur le lecteur ou sur le copiste. Elle est plutôt la priorité d'une règle sur l'activité qu'elle gouverne. (1996, p. 333).

De là, ainsi que j'ai pu précédemment le suggérer, l'urgence d'inscrire la question des transformations des modes de régulation et, plus généralement, celle des nouvelles rationalisations institutionnelles à l'agenda des sciences sociales.

Je tire de cette remarque une conclusion d'ordre épistémologique: si elle possède de solides fondements ontologiques, la vieille dichotomie erklären/verstehen (dont le triptyque habermassien n'est qu'une déclinaison raffinée) mérite assouplissement. Comment en effet contribuer à l'intelligence des modes d'institution du sens, être capable de comprendre les logiques et les principes qui gouvernent les actions et les représentations... sans, dans le même temps, pouvoir expliquer le poids des (in)cohérences systémiques qui informent les mondes vécus? J'ai conscience, disant cela, d'énoncer une évidence sociologique que, dès les premières pages d'Economie et société, M. Weber s'était fait fort de conceptualiser. Il faut reconnaître cependant qu'à force d'hypertrophier la démarche compréhensive de $\mathrm{M}$. Weber, nombre de commentaires ont fini par durcir à l'excès une disjonction qui, dans la pratique des sciences sociales, est beaucoup moins décisive qu'on ne le croit parfois. Ces considérations ne sont-elles pas après tout qu'antiennes épistémologiques? Pour revenir à notre questionnement initial, ne faut-il pas plutôt prêter attention au statut du discours des sciences sociales à l'heure actuelle? Si tel est le cas, il est intéressant de rappeler le statut spécifique de la sociologie depuis sa naissance: celle-ci est à la fois le produit et le moyen d'escorte de la modernité (Wagner, 1996). Le basculement auquel nous assistons aujourd'hui ne fait qu'accentuer la singularité de notre discipline. Mais 
il en sape également quelques fondements majeurs. A. Giddens ne dit rien d'autre en écrivant que

\begin{abstract}
la modernité est profondément et intrinsèquement sociologique. Une part importante de l'inconfort du sociologue professionnel, en tant que le pourvoyeur d'un savoir expert sur la vie sociale, vient du fait qu'il ne devance guère les praticiens profanes éclairés de la discipline (1994, p. 49).
\end{abstract}

Il faut tirer les conséquences d'une telle affirmation: puisque la production d'un savoir «savant» n'est plus l'apanage des seuls scientifiques labellisés comme tels, ne faut-il pas œuvrer plus activement que jamais en faveur d'une réappropriation à la fois critique et démocratique des connaissances que nous livrons ${ }^{11}$ ? Plus important encore: outre la mise au jour des modes de production du sens, les sciences sociales n'ont-elles pas aussi pour tâche désormais de contribuer activement et ouvertement au procès d'institution lui-même? Ma réponse est affirmative. Offrir des réservoirs de sens dans lesquels puissent puiser les individus et les groupes est une manière de lier autrement sociologie et morale, et cela sans pour autant céder le moindre pouce sur le terrain de la scientificité ${ }^{12}$.

En tant qu'orogénie du monde social, la sociologie ne doit pas qu'expliquer les multiples plis, failles et dislocations dont sont pétries les sociétés contemporaines. Débitrice de l'exigence démocratique qui l'a tôt configurée, la sociologie est et doit d'abord demeurer un instrument réflexif au service de l'autonomie collective et individuelle. A condition de toujours maîtriser au plus près les réalités du terrain de la quotidienneté, elle doit ensuite offrir les moyens d'interpréter et de vivre les destins personnels autrement que sur le mode tragique de la culpabilisation et du nihilisme destructeur. En oeuvrant de la sorte au service d'une politique du sens, la sociologie contribue à la production de ce «frêle bonheur» qu'évoque si bien J. J. Rousseau dans son Emile. Mais, depuis les réflexions de l'auteur du Contrat social sur les fondements de notre identité moderne, les temps ont changé. Les reconfigurations institutionnelles de ces dernières décennies ont précipité la dislocation des cohérences identitaires (Dubar, 2000) et, avec elles, celles des anciennes partitions épistémologiques. Voilà pourquoi, à l'heure de la recomposition des modes d'institution du social, la sociologie doit satisfaire un double impératif. Il s'agit d'abord de maintenir l'institution comme point de mire analytique prioritaire, et cela pour mieux penser les aventures d'un sujet qui, pas

11 De manière concrète une telle posture invite à assortir notre travail académique d'un souci de vulgarisation intelligente de ce que peuvent produire et dire les sciences sociales contemporaines et surtout à ne pas se laisser piéger par un scientisme vulgaire qui, au nom de la complexité du monde, récuse tout effort de démocratisation des savoirs. Il est vrai, je l'ai précisé précédemment, que le savoir sur nous mêmes n'est nullement un gage définitif d'émancipation. Cette autoconnaissance partagée et éclairée restera par ailleurs bien mal partagée tant que l'accès aux choses de la culture restera grevé d'iniquités. Il s'agit donc avant tout de viser un idéal dont la vertu première est de participer à la déculpabilisation individuelle et collective et non d'adhérer naïvement aux vertus présupposées des discours pédagogiques.

12 Je ne reviens pas, à ce stade de l'exposé, sur le problème des critères de scientificité des sciences sociales contemporaines. Sans pour autant placer ses propos sous les auspices de l'approche habermassienne mobilisée pour la présente contribution, J.C. Passeron (1991) a montré de façon convaincante que le raisonnement sociologique s'inscrit dans un espace qui échappe aux critères de l'épistémologie poppérienne. 
plus qu'hier, n'échappe à la pesanteur des mondes sociaux qu'il habite. Il s'agit ensuite de renoncer à la pureté de l'idéal praxéologique qui n'a plus lieu d'être aujourd'hui. A la condition néanmoins de garder vivace l'exigence critique qui l'a vu naître, la sociologie peut alors s'avérer une science précieuse pour que chacun d'entre nous puisse s'affirmer en toute plénitude citoyen et travailleur, homo politicus et animal laborans, responsable du monde et acteur de sa vie..., bref, héros et anti-héros.

\section{Analyse sociologique du travail, de l'emploi et des organisations Conservatoire National des Arts et Métiers, Paris}

\section{RÉFÉRENCES BIBLIOGRAPHIQUES}

T. Adorno, K. Popper, De Vienne à Francfort. La querelle allemande des sciences sociales, Bruxelles, Complexe, 1979 [Edition allemande originale: 1969].

H. Albert, La sociologie critique en question, Paris, PUF, 1987.

K. O. Apel, Expliquer-comprendre, Paris, Cerf, 2000 [Edition originale: 1979].

S. Beaud, M. Pialloux, Retour sur la condition ouvrière, Paris, Fayard, 1999.

U. Beck, La société du risque, Paris, Aubier, 2001 [Edition originale: 1986].

N. Beckenbach, Industriesoziologie, Berlin, New York, Walter de Gruyter, 1991.

P. Bernoux, «L'entreprise est-elle toujours une institution?», Sociologie du travail, vol. 41, 1999, n 2 , pp. 179-194.

D. Bertaux, Les récits de vie, Paris, Nathan, 1997.

L. Boltanski, E. Chiapello, Le nouvel esprit du capitalisme, Paris, Gallimard, 1999.

P. Bourdieu, «La représentation politique. Eléments pour une théorie du champ politique », Actes de la recherche en sciences sociales, février-mars 1981, n³6/37, p. 3-24.

R. Boyer, M. Freyssenet, Les modèles productifs, Paris, La Découverte, 2000.

P. Cahuc, A. Zylberberg, Microéconomie du marché du travail, Paris, La Découverte, 2003.

R. Castel, Les métamorphoses de la question sociale. Une chronique du salariat, Paris, Fayard, 1995.

J. De Munck, «Les métamorphoses de l'autorité », Autrement, 2000, n 198, pp. 21-42.

D. Demazière, C. Dubar, Analyser les entretiens biographiques. L'exemple de récits d'insertion, Paris, Nathan, 1997.

V. Descombes, Les institutions du sens, Paris, Minuit, 1996.

C. Dubar, La crise des identités, Paris, PUF, 2000.

F. Dubet, Le déclin de l'institution, Paris, Seuil, 2002.

J. P. Durand, P. Stewart, J. J. Castillo (éds), L'avenir du travail à la chaîne, Paris, La Découverte, 1998.

E. Durkheim, Pragmatisme et sociologie, Paris, Vrin, 1955.

A. Ehrenberg, La fatigue d' être soi, Paris, Odile Jacob, 1998.

E. Enriquez, «L'entreprise comme lien social: un colosse aux pieds d'argile» in R. Sainsaulieu (éd), L'entreprise, une affaire de société, Paris, Presses de la FNSP, 1990, pp. 203-228.

F. Ferrarotti, Histoire et histoires de vie. La méthode biographique dans les sciences sociales, Paris, Méridiens-Klincksieck, 1983.

P. Feyerabend, La science en tant qu' art, Paris, Albin Michel, 2003.

M. Foucault, «La psychologie de 1850 à 1950 », in D. Huisman, A. Weber (éds), Histoire de la philosophie européenne, t. II, Tableau de la philosophie contemporaine, Paris, Fischbacher, 1957, pp. 591-606. 
E. Friedberg, «Faire son métier de sociologue, surtout dans l'intervention» in D. Vrancken, O. Kuty (éds), La sociologie et l'intervention, Bruxelles, De Boeck Université, 2001, pp. 111-130.

B. Gazier, Economie du travail et de l'emploi, Paris, Dalloz, 1991.

A. Giddens, Les conséquences de la modernité, Paris, L'Harmattan, 1994.

J. Habermas, La technique et la science comme «idéologie», Paris, Gallimard, 1973 [Edition originale: 1968].

J. Habermas, Connaissance et intérêt, Paris, Gallimard, 1976 [Edition originale: 1968].

J. Habermas, «Trente ans plus tard: remarques sur Connaissance et intérêt», in E. Renault, Y. Sintomer (éds), Où en est la théorie critique? Paris, La Découverte, 2003, pp. 93-100.

A. Honneth, La lutte pour la reconnaissance, Paris, Cerf, 2000.

M. Horkheimer, Théorie critique, Paris, Payot, 1978.

E. Husserl, La crise des sciences européennes et la phénoménologie transcendantale, Paris, Gallimard, 1976 [Edition originale: 1954].

J. C. Kauffman, «L'expression de soi », Le débat, n 119, mars-avril 2002, p. 116-125.

B. Lahire (ed), A quoi sert la sociologie? Paris, La Découverte, 2002.

M. Lallement, «Les transformations des relations professionnelles en France: éléments pour une mise en perspective à l'heure de la Refondation sociale », Schweizerische Zeitschrift für Soziologie, vol. $28,2002, \mathrm{n}^{\circ} 3$, pp. 453-474.

D. Linhart, La modernisation des entreprises, Paris, La Découverte, 1994.

N. Luhmann, La légitimation par la procédure, Presses de l'université de Laval, Paris, Cerf, 2001 [Edition originale: 1969].

S. Mallet, La nouvelle classe ouvrière, Paris, Seuil, 1963.

N. Murard, La morale de la question sociale, Paris, La dispute, 2003.

C. Offe, «Le travail comme catégorie de la sociologie», Les temps modernes, 1985, n 466, pp. 20582094.

J. C. Passeron, Le raisonnement sociologique. L'espace non-poppérien du raisonnement naturel, Paris, Nathan, 1991.

T. Pillon, F. Vatin, Traité de sociologie du travail, Toulouse, Octares éditions, 2003.

A. Pouchet (éd), Sociologies du travail : 40 ans après, Paris, Elsevier, 2001.

J. D. Reynaud, Les règles du jeu. L'action collective et la régulation sociale, Paris, Colin, 1989.

P. Ricœur, Soi-même comme un autre, Paris, Seuil, 1990.

G. Rot, «Autocontrôle, traçabilité, responsabilité», Sociologie du travail, vol. XL, 1998, n 1, pp. 520.

R. Sainsaulieu, D. Segrestin, «Vers une théorie sociologique de l'entreprise», Sociologie du travail, vol. XXVIII, 1986, n³, pp. 335-352.

A. Supiot, «La contractualisation de la société», conférence publique à l'université de tous les savoirs, Paris, CNAM, 22 février 2000, miméo., 11 pages.

C. Taylor, Les sources du moi, Paris, Seuil, 1998 [Edition originale: 1989].

A. Touraine, La conscience ouvrière, Paris, Seuil, 1966.

P. Wagner, Liberté et discipline. Les deux crises de la modernité, Paris, Métailié, 1996 [Edition originale: 1995$]$

B. Walliser (1989), «Théorie des jeux et genèse des institutions», Recherches économiques de Louvain, 55 (4), pp. 339-364.

H. Zajdela, «Que nous apprend la nouvelle économie du travail?», La revue du MAUSS, 1994, n 3, $\mathrm{n}^{\circ}$ spécial «Pour une autre économie», Paris, La Découverte, pp. 60-76.

P. Zarifian, «L'agir communicationnel face au travail professionnel», Sociologie du travail, vol. 41, $1999, \mathrm{n}^{\circ} 2$, pp. 163-177. 